\section{Safety of Radial Arterial Catheterization in PET Research Subjects}

TO THE EDITOR: Arterial blood sampling is used in PET to determine metabolite-corrected arterial input functions. These arterial blood samples are collected through a catheter placed percutaneously in the radial artery at the wrist. Indwelling arterial catheters are typically used for continuous hemodynamic monitoring of critically ill patients, and the risk of thrombotic and ischemic complications in such clinical populations is reported to be low (1-3). However, few published data deal directly with the potential adverse effects of radial arterial catheterization in physically healthy PET research subjects, in whom the relative risks and benefits of the procedure are very different from those in clinical settings.

Our group followed a total of 1,132 radial arterial cannulations in 924 PET research subjects (530 men [average age, $42.9 \pm$ $16.59 \mathrm{y}$ ] and 340 women [average age, $43.5 \pm 17.10 \mathrm{y}$ ]; sex data not available for 54 subjects). Under certain PET protocols, scans were performed before and after 4-8 wk of psychiatric treatment; as a result, 208 subjects were catheterized twice. Data were obtained from PET studies conducted by the Division of Biologic Psychiatry, the Division of Translational Imaging, and the Division of Molecular Imaging.

Before enrollment in PET research protocols, all subjects were screened to confirm normal blood hemoglobin levels (minimum, 11-14 g/dL) and adequate collateral ulnar circulation (normal results on Allen test). No subjects had sustained recent wrist trauma. Experienced physicians percutaneously placed radial arterial catheters (18-20 gauge) at a site prepared with alcohol and subcutaneous lidocaine $(1.0 \%-2.0 \%)$. Between 117 and $137 \mathrm{~mL}$ of blood were taken during PET scans. On average, catheters were in place for $5 \mathrm{~h}$ (range, $1-7 \mathrm{~h}$ ).

After decannulation, a physician applied pressure directly to the site for a minimum of $10 \mathrm{~min}$. Subjects were given a pressure dressing and instructions to contact an on-call physician regarding any pain or abnormality in the hand, wrist, or arm during the subsequent days. Follow-up appointments were scheduled within a month of catheterization, at which time physicians examined subjects' wrists (56\% of patients kept these appointments). If subjects failed to keep this appointment, repeated efforts were made to contact them via phone and mail.
Of 1,132 arterial lines in 924 subjects, there was 1 instance of symptomatic thrombotic occlusion $(0.09 \%)$, documented by Doppler ultrasound in a depressed female patient. There was no associated ischemic damage, and the condition resolved over a period of weeks without intervention. Our rate of thrombotic occlusion is significantly lower than previously published results, which range from $9.8 \%$ to $25 \%(1-3)$. This difference may be due to differences in patient populations, as subjects in our cohort were at lower risk for circulatory complications.

We conclude that short-term arterial cannulation rarely results in clinically relevant adverse events and that it is safe and appropriate for use in physically healthy research subjects, about whom it can provide important data.

\section{REFERENCES}

1. Scheer BV, Perel A, Pfeiffer UL. Complications and risk factors of peripheral arterial catheters used for haemodynamic monitoring in anaesthesia and intensive care medicine. Crit Care. 2002;6:198-204.

2. Slogoff S, Keats AS, Arlund C. On the safety of radial artery cannulation. Anesthesiology. 1983;59:42-47.

3. Weiss BM, Gattiker RI. Complications during and following radial artery cannulation: a prospective study. Intensive Care Med. 1986;12:424-428.

\section{Benjamin A. Everett* \\ Maria A. Oquendo \\ Anissa Abi-Dargham \\ Mitchell S. Nobler \\ Davangere P. Devanand \\ Sarah H. Lisanby \\ J. John Mann \\ Ramin V. Parsey}

*Columbia University College of Physicians and Surgeons

630 W. 168th St.

Mailbox 541

New York, NY 10032

E-mail: benjamin.everett@gmail.com

DOI: $10.2967 /$ jnumed.109.063206

COPYRIGHT @ 2009 by the Society of Nuclear Medicine, Inc. 